EESTI NSV TEADUSTE AKADEEMIA TOIMETISED, 29. KOIDE FUOSIKA * MATEMAATIKA. 1980, NR. 1

ИЗВЕСТИЯ АҚАДЕМИИ НАУК ЭСТОНСКОИ ССР. ТОМ 29 ФИЗИКА * МАТЕМАТИКА, 1980, № 1

И. КЕИС

удК $62.5 \mathrm{U}$

\title{
СУБОПТИМАЛЬНЫЙ СИНТЕЗ УПРАВЛЕНИЯ МЕТОДОМ ОБРАЩЕНИЯ И ПСЕВДОАВТОНОМНОСТИ
}

I. KEIS. JUHTIMISE SUBOPTIMAALNE SONTEES POORD- JA PSEUDOAUTONOOMSUSMEETODIL

1. KEIS. A SYNTHESIS OF SUBOPTIMAL CONTROL VIA INVERSE AND PSEUDOAUTONOMOUS METHODS

(Представлена Н. Алумяэ)

В работе продолжено построение регуляторов сложных систем $\left[{ }^{1-3}\right]$. Уравнения возмущений $x \equiv 0$ стабилизируемой по $x^{1}, x^{\prime}$ системы

$$
\begin{gathered}
x=F(t, x, u), \quad 0 \leqslant t, \quad x \in R=\left\{\left|x^{1}\right| \leqslant h_{0}\right\}=R_{0} \cup x^{\prime}=0, \\
u=\left(u_{\sigma}\right)^{*} \in \Omega=\left\{g(u) \leqslant g_{0}\right\},
\end{gathered}
$$

$$
\begin{gathered}
x^{1}=\left(x_{i}\right)^{*}, \quad x^{\prime}=\left(x_{j}\right)^{*}, \quad x=\left(x_{i}, x_{v}\right)^{*} \quad\left(i=\overline{1, m^{1}}, \quad j=\overline{1, m^{\prime}}, \quad v=\overline{m^{1}+1, n,}\right. \\
\left.\sigma=\overline{1, r,} m^{\prime} \leqslant m^{1}\right)
\end{gathered}
$$

с целью управления $\left\{x^{\prime}=0\right\}$, нормой $\left.g(u)(g \in G){ }^{1}\right]$ и критерием

$$
I=\int_{t}^{t_{1}} F_{0}(\tau, x, u) d \tau \rightarrow \min _{u}\left(x^{\prime} \rightarrow 0, t \rightarrow t_{1}-0, t_{1}=\min t_{1}^{\prime} ; \quad u, F, F_{0} \in C\left(R_{0}\right)\right)
$$

рассмотрим в агрегирующих [2] переменных $y=\left(y_{k}(t, x, \alpha)\right)$, $z=\left(z_{s}\right)^{*}$

$y=Y(t, \xi, u, \alpha), \quad z=Z(t, \xi, u, \alpha), \quad D_{0}=\xi\left(R_{0}\right)=D \backslash y^{\prime}=0\left(\xi=\left(y_{k}, z_{s}\right)^{*}\right)$,

$$
\begin{gathered}
I=\int_{t}^{t_{1}} f_{0} d \tau \rightarrow \min _{u}, \quad f_{0}=\left.F\right|_{x=x(\xi)}, \quad \alpha=\left(\alpha_{\mu}\right)^{*}=\mathrm{const} \in \Delta_{1}\left(0, \varrho_{1}\right) \in E^{m_{\mathrm{t}}}, \\
y^{\prime}=\left(y_{q}\right)^{*}, y^{\prime \prime}=\left(\dot{y}_{\beta}\right)^{*}, z=\left(z_{s}\right)^{*}\left(q=\overline{1, l_{1}}, \beta=\overline{l_{1}+1, l, \mu}=\overline{1, m_{1}}, l \ll n\right),
\end{gathered}
$$

где $\forall \varepsilon \leqslant d_{0}\left(h_{0}, \alpha\right) \exists \delta\left(t_{0}, z_{0}, \alpha, \varepsilon\right):|y|<\varepsilon,\left[t_{0}, t_{1}\right) ; \quad y^{\prime} \rightarrow 0, \quad t \rightarrow t_{1}, \quad\left|y_{0}\right| \leqslant \delta$. Агрегаты $y$ и субоптимальные сгабилизаторы $u^{\prime}$ системы (1.1) функции $t$, измеряемых величин $h_{k}(t, x)(k=\overline{1, l})$ и вектора агрегации $\alpha$. Из оптимальной $y, y^{\prime}$-устойчивости $(1.2)$ в области $P(\alpha)=$ $=\left\{y^{\prime} \neq 0,|y| \leqslant \delta(\alpha)\right\}$ при всяком $u^{\prime} \in\left\{u^{\prime}\right\}=U^{\prime}$ имеем $\left[{ }^{2,3}\right]$ оптимальную $x^{1}, x^{\prime}$-стабилизацию (1.1) на $Q(\alpha)=x(P(\alpha)) \subseteq R_{0}$.

1. Способ обращения производящим потенциалом $S^{+}(t, y, \alpha, \gamma)$. Пусть для $S^{+} \in C\left(D^{\prime}\right), \quad D^{\prime}=\left\{D \times \Delta_{1} \times \Delta_{2}\right\}=$ $\left.=D_{0}^{\prime} \cup y^{\prime}=0\left(\gamma=\gamma_{\lambda}\right)^{*} \in \Delta_{2}\left(0, \varrho_{2}\right), \quad \lambda=\overline{1, m_{2}}\right) \quad S^{+} \equiv 0, d S^{+} / \partial y^{\prime \prime} \equiv 0$ 
$\left(y^{\prime}=0\right) ; S^{+} \in C_{1}\left(D_{0}^{\prime}\right)$ существует и является единственной седловая точка $z^{+}, u^{+}$гамильтониана системы (1.2)

$$
H^{+}\left(z^{+}, u\right) \geqslant H^{+}\left(z^{+}, u^{+}\right)=\max _{z} \min _{u} H^{+}(z, u) \geqslant H^{+}\left(z, u^{+}\right)\left(H^{+} \equiv H\left[S^{+}\right]\right),
$$

$u^{+}=u^{+}(t, y, \alpha, \gamma) \in C\left(D_{0}^{\prime}\right), \quad u^{+} \in \Omega, \quad z^{+}=z^{+}(t, y, \alpha, \gamma) \subset C\left(D_{0}^{\prime}\right), \quad z^{+} \in D_{0}^{\prime}$, $H\left[S^{+}\right]=\partial S^{+} / \partial t+Y \cdot p^{+}+f_{0}, \quad p_{0}^{+}=\partial S^{+} / \partial t, \quad p^{+}=\partial S^{+} / \partial y$,

$$
H^{+}\left(z^{+}, u^{+}\right) \equiv-h_{+}(t, y, \alpha, \gamma),
$$

где вектор-функция $u^{+}(t, y, \alpha, \gamma)$ - стабилизатор системы (1.2), т. е. $u^{+} \in U^{\prime}$. Обозначим через $U^{\prime \prime}=\left\{u^{\prime \prime}\right\}$ множество стабилизаторов агрегированной (1.2). Из (1.3) следует, что регулятор $u^{+}-$субоптимальный $\left[{ }^{2,3}\right]$ для (1.2), если $U^{\prime \prime} \ni u^{+}(t, y, \alpha, \gamma)$ - стабнлизатор агрегированной из (1.2) системы

$$
\begin{gathered}
y_{+}=Y\left(t, y, z^{+}, u, \alpha\right), \quad I_{+}=\int_{t}^{t_{+}} f_{+} d \tau \rightarrow \min _{u},\left.\quad f_{+} \equiv f_{0}\right|_{z=z^{+}}+h_{+}, \\
S^{+}=\min _{u} I_{+}=I_{+}\left(u^{+}\right)=\int_{t}^{t_{+}} f_{+}^{+} d \tau\left(\left.f_{+}^{+} \equiv f_{+}\right|_{u=u^{+}}, h_{+} \equiv-H^{+}\left(z^{+}, u^{+}\right)\right) .
\end{gathered}
$$

При этом $S^{+}, u^{+}[t], z^{+}[t]-$ решение задачи оптимальной стабилизации

$$
\begin{gathered}
y^{*}=Y(t, \xi, u, \alpha), \quad S^{+}=\max _{z} \min _{u} I_{+}^{\prime}, \quad u \in \Omega, \quad z \in D_{0}\left(I_{+}^{\prime}=\int_{t}^{t^{\prime}}\left(f_{0}+h_{+}\right) d \tau\right), \\
S^{+} \geqslant \min _{u} I_{+}^{\prime}(z, u)\left(z, u \in C\left[t_{0}, t^{\prime}\right), t^{\prime}: y^{\prime} \rightarrow 0, t \rightarrow t^{\prime}-0\right),
\end{gathered}
$$

где наихудшая по $I_{+}^{\prime}$ стратегия $z^{+}$вообще не $z[t]$-компонента решения (1.2). Интегрируя величину $h_{+}+H^{+}\left(u^{+}, z\right)$ вдоль (1.2) при $u=$ $=u^{+} \in U^{\prime} \cap U^{\prime \prime}$, находим из (1.3) для субоптимальной функции $I\left(u^{+}\right) \equiv I^{+}(t, \xi, \alpha, \gamma)$ оценки

$$
\begin{gathered}
I^{+}=\int_{t}^{t^{+}}\left(\left.f_{0}\right|_{u=u^{+}}+h_{+}\right) d \tau+\int_{t}^{t^{+}} H^{+}\left(z^{+}, u^{+}\right) d \tau \leqslant S+-\int_{t}^{t^{+}} h_{+} d \tau=\int_{t}^{t^{+}} f_{0}\left(z^{+}, u^{+}\right) d \tau, \\
I^{+} \leqslant S^{+}(t, y, \boldsymbol{\alpha}, \gamma), \text { если } H^{+}\left(z^{+}, u^{+}\right) \leqslant 0 ; t^{+}=\min t^{*}: y^{\prime} \rightarrow 0, t \rightarrow t^{*} ;
\end{gathered}
$$

$$
H_{+} \equiv H^{+}(z, u)+h_{+} .
$$

Оптимальный по $I^{+}$параметр $\gamma^{0}$ определим $\forall t_{0}, y_{0}, z_{0}, \alpha$ условиями $\left[{ }^{3}\right]$, введя вектор $a=\left(a_{\mu}, \gamma_{\lambda}\right)^{*} \quad\left(\xi_{0}=\xi\left[t_{0}\right], \mu=\overline{1, m_{1}}, \lambda=\overline{1, m_{2}}\right)$.

Условия оптимальности $\gamma^{0}$ упрощаются, если вместо точечной минимизации по критериям близости $I^{+}$к $I_{\text {opt }}\left[{ }^{2,3}\right]$ найти $\gamma^{0}$ из требования локального минимума нормы $f_{0}\left(z^{+}, u^{+}\right)$или $S^{+}(t, y, \alpha, \gamma)$ на $t \geqslant 0$, $|y| \leqslant \delta \leqslant d_{0}, \forall \alpha \in \Delta_{1}$ в мажорантах (1.4). Выберем $\gamma^{0}$. Тогда оптимизация по $\alpha$ субоптимального регулятора $u^{+}\left(t, y, \alpha, \gamma^{0}\right)$, заданного в (1.3) потенциалом $S^{+}$при условиях (1.3), проводится согласно схеме [3] из условий теоремы Болтянского. Наилучший субоптимальный стабилизатор $u_{0}^{+}$системы (1.1) находим из $u^{+}$подстановкой $y=$ $=y\left(t, x, \alpha^{0}, \gamma^{0}\right)$, где $\alpha^{0}=\alpha_{\text {opt }}=\alpha^{0}\left(t, \xi, \gamma^{0}\right)$. 
2. Способ псевдо автономности по $y$ в агрегировании по функционалу. Предположим, что функции $Y, f_{0}$ представимы на $D_{0}$ в виде

$$
\begin{gathered}
Y=Y_{1}^{0}(t, y, \alpha)+A_{1}(t, y, \alpha) u+Y_{2}^{0}(t, \xi, \alpha)+A_{2}(t, \xi, \alpha) u+Y_{2}(t, \xi, u, \alpha) \\
f_{0}=f_{1}^{0}(t, y, \alpha)+a_{1}(t, y, \alpha) \cdot u+f_{2}^{0}(t, \xi, \alpha)+a_{2}(t, \xi, \alpha) \cdot u+f_{2}(t, \xi, u, \alpha) \\
\left.y_{2}^{0}\right|_{z=0} \equiv 0,\left.\quad f_{2}^{0}\right|_{z=0} \equiv 0,\left.\quad A_{2}\right|_{z=0} \equiv 0,\left.\quad a_{2}\right|_{z=0} \equiv 0 \\
\quad Y_{2}=|u| E_{2}(\cdot \mid u), \quad f_{2}=|u| \varepsilon_{2}(\cdot \mid u), \\
b \equiv A_{1}^{*} p+a_{1} \neq 0, \quad E_{2} \rightarrow 0, \quad|u| \rightarrow 0, \quad \varepsilon_{2} \rightarrow 0, \quad|u| \rightarrow 0\left(p_{0}=\partial S^{\prime} / \partial t, \quad p=\partial S^{\prime} / \partial y\right), \\
\quad A_{1}=\left\|a_{k \sigma}^{1}\right\|, A_{2}=\left\|a_{k \sigma}^{(2)}\right\|, a_{1}=\left(a_{\sigma}^{1}\right)^{*}, a_{2}=\left(a_{\sigma}^{(2)}\right)^{*} \quad(k=\overline{1, l} \ll n, \sigma=\overline{1, r)},
\end{gathered}
$$

где все элементы разложения (2.1) имеют необходимую гладкость. Агрегируя лишь по функционалу $(1.2)$, заменим $f_{0}$ на новое ядро

$$
\begin{gathered}
f_{0}^{\prime}=f_{0}+h^{\prime}, \\
I^{\prime}=\int_{t}^{t_{1}^{\prime}} f_{0}^{\prime} d \tau\left(-h^{\prime}(t, \xi, u, \alpha) \equiv f_{2}^{0}+p \cdot Y_{2}^{0}+\left(a_{2}+A_{2}^{*} p\right) \cdot u+p \cdot Y_{2}+f_{2}\right) .
\end{gathered}
$$

Гамильтониан системы $(1.2),(2.1),(2.2)$ с функционалом $I^{\prime}-$ $t, y$-автономная, линейная по $u$ функция $H^{\prime}\left(t, y, p_{0}, p, u, \alpha\right)$ вида

$$
H^{\prime} \equiv H\left[S^{\prime}\right]=\partial S^{\prime} / \partial t+f_{1}^{0}+p \cdot Y_{1}^{0}+b \cdot u\left(y \in D_{0}, u \in \Omega, b=A_{1}^{*} p+a_{1} \neq 0\right)
$$

- достигает абсолютного минимума $H^{\prime}=0$ на экстремальном регуляторе

$$
\begin{aligned}
& u^{\prime}=-g_{0} \partial g^{\prime} / \partial b, g^{\prime}(u)=\max _{g(v)=1}(v \cdot u) \quad\left(g>0, v \neq 0, g^{\prime}>0, u \neq 0,\right. \\
& g(u)=g(-u)) \text {, } \\
& g^{\prime} \in G(u): g^{\prime}(\lambda u)=|\lambda| g^{\prime}(u), g^{\prime}\left(u_{2}\right)-g^{\prime}\left(u_{1}\right)>\nabla g^{\prime}\left(u_{1}\right) \cdot\left(u_{2}-u_{1}\right) \\
& \left|u_{1}\right|\left|u_{2}\right|>\left|\left(u_{1} \cdot u_{2}\right)\right| \text {, }
\end{aligned}
$$

где функция $S^{\prime}$ определена уравнением порядка $l \ll n$ и условиями $\partial S^{\prime} / \partial t+f_{1}^{0}(t, y, \alpha)+p \cdot Y_{1}^{0}(t, y, \alpha)-g_{0} g^{\prime}\left(A_{1}^{*} p+a_{1}\right)=0 ; \quad S^{\prime} \equiv 0, \quad \partial S^{\prime} / \partial y^{\prime \prime} \equiv 0$

$$
\left(y^{\prime}=0\right) \text {. }
$$

Пусть (2.3) - стабилизатор системы (1.2). Тогда $u^{\prime}(t, y, \alpha)-$ субоптимальный регулятор (1.2), так как $u^{\prime} \in U^{\prime} \cap U^{\prime \prime}$. Вид функции $u^{\prime}$ в (2.3) устанавливается решением (2.4). Минимизацию субоптимальной функции $I\left(u^{\prime}\right)$ по вектору агрегации $\alpha$ с целью поиска наилучшего $\alpha^{0}$ можно проводить аналогично $\left[{ }^{3}\right]$.

\section{ЛИ Т Е Р А Т У Р А}

1. Ке й с И., Изв. АН ЭССР, Физ. Матем., 26, № 1, 37-47 (1977).

2. Ке й с И., Изв. АН ЭССР, Физ. Матем., 27, № 3, 274-288 (1978).

3. К е й с И., Изв. АН ЭССР, Фнз. Матем., 28, № 2, 107-114 (1979).

Институт кибернетики Академии наук Эстонской ССР
Поступила в редакцию 2/VII 1979 